\title{
Random sampling causes the low reproducibility of rare eukaryotic OTUs in Illumina COI metabarcoding
}

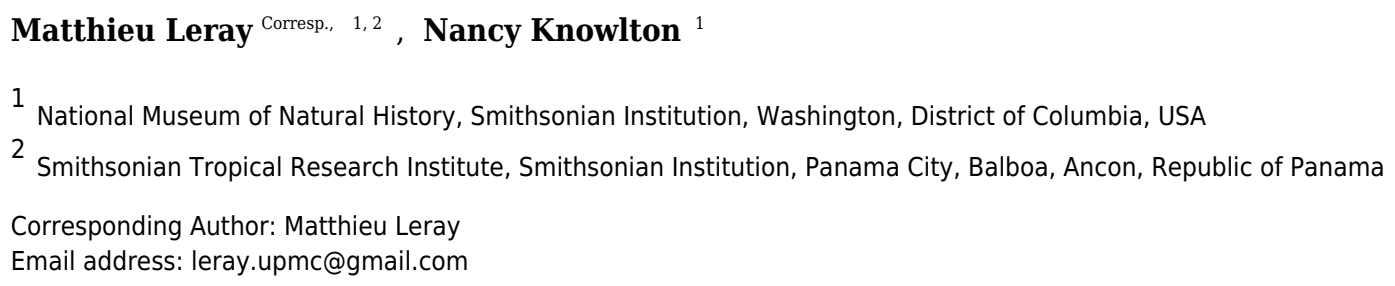

DNA metabarcoding, the PCR-based profiling of natural communities, is becoming the method of choice for biodiversity monitoring because it circumvents some of the limitations inherent to traditional ecological surveys. However, potential sources of bias that can affect the reproducibility of this method remain to be quantified. The interpretation of differences in patterns of sequence abundance and the ecological relevance of rare sequences remain particularly uncertain. Here we used one artificial mock community to explore the significance of abundance patterns and disentangle the effects of two potential biases on data reproducibility: indexed PCR primers and random sampling during Illumina MiSeq sequencing. We amplified a short fragment of the mitochondrial Cytochrome c Oxidase Subunit I (COI) for a single mock sample containing equimolar amounts of total genomic DNA from 34 marine invertebrates belonging to six phyla. We used seven indexed broad-range primers and sequenced the resulting library on two consecutive Illumina MiSeq runs. The total number of OTUs was $\sim 4$ times higher than expected based on the composition of the mock sample. Moreover, the total number of reads for the 34 components of the mock sample differed by up to three orders of magnitude. However, 79 out of 86 of the unexpected OTUs were represented by $<10$ sequences that did not appear consistently across replicates. Our data suggest that random sampling of rare OTUs (e.g. small associated fauna such as parasites) accounted for most of variation in OTU presence-absence, whereas biases associated with indexed PCRs accounted for a larger amount of variation in relative abundance patterns. These results suggest that random sampling during sequencing leads to the low reproducibility of rare OTUs. We suggest that the strategy for handling rare OTUs should depend on the objectives of the study. Systematic removal of rare OTUs may avoid inflating diversity based on common $\beta$ descriptors but will exclude positive records of taxa that are functionally important. Our results further reinforce the need for technical replicates (parallel PCR and sequencing from the same sample) in metabarcoding experimental 
designs. Data reproducibility should be determined empirically as it will depend upon the sequencing depth, the type of sample, the sequence analysis pipeline, and the number of replicates. Moreover, estimating relative biomasses or abundances based on read counts remains elusive at the OTU level. 
1 Random sampling causes the low reproducibility of rare eukaryotic OTUs in Illumina COI

2 metabarcoding

3

4 Matthieu Leray $^{1,2 *}$ and Nancy Knowlton ${ }^{1}$

5

$6{ }^{1}$ National Museum of Natural History, Smithsonian Institution, Washington, District of

7 Columbia, USA

8 2Smithsonian Tropical Research Institute, Smithsonian Institution, Panama City, Balboa, Ancon,

9 Republic of Panama

10

$11 *$ corresponding author, E-mail: leray.upmc@gmail.com

12

13 
14 ABSTRACT

15

DNA metabarcoding, the PCR-based profiling of natural communities, is becoming the method of choice for biodiversity monitoring because it circumvents some of the limitations inherent to traditional ecological surveys. However, potential sources of bias that can affect the reproducibility of this method remain to be quantified. The interpretation of differences in patterns of sequence abundance and the ecological relevance of rare sequences remain particularly uncertain.

Here we used one artificial mock community to explore the significance of abundance patterns and disentangle the effects of two potential biases on data reproducibility: indexed PCR primers and random sampling during Illumina MiSeq sequencing. We amplified a short fragment of the mitochondrial Cytochrome c Oxidase Subunit I (COI) for a single mock sample containing equimolar amounts of total genomic DNA from 34 marine invertebrates belonging to six phyla. We used seven indexed broad-range primers and sequenced the resulting library on two consecutive Illumina MiSeq runs.

The total number of OTUs was $\sim 4$ times higher than expected based on the composition of the mock sample. Moreover, the total number of reads for the 34 components of the mock sample differed by up to three orders of magnitude. However, 79 out of 86 of the unexpected OTUs were represented by $<10$ sequences that did not appear consistently across replicates. Our data suggest that random sampling of rare OTUs (e.g. small associated fauna such as parasites) accounted for most of variation in OTU presence-absence, whereas biases associated with indexed PCRs accounted for a larger amount of variation in relative abundance patterns. These results suggest that random sampling during sequencing leads to the low reproducibility of rare OTUs. We suggest that the strategy for handling rare OTUs should depend on the objectives of the study. Systematic removal of rare OTUs may avoid inflating diversity based on common $\beta$ descriptors but will exclude positive records of taxa that are functionally important. Our results further reinforce the need for technical replicates (parallel PCR and sequencing from the same sample) in metabarcoding experimental designs. Data reproducibility should be determined empirically as it will depend upon the sequencing depth, the type of sample, the sequence analysis pipeline, and the number of replicates. Moreover, estimating relative biomasses or abundances based on read counts remains elusive at the OTU level. 
47

48

49

50

51

52

53

54

55

56

57

58

59

60

61

62

63

64

65

66

67

68

69

70

\section{INTRODUCTION}

High-throughput sequencing of short homologous DNA fragments from mixed bulk samples, an approach referred to as DNA metabarcoding, has become very popular for monitoring diversity because it circumvents limitations inherent to morphological identifications (Taberlet et al. 2012; Ji et al. 2013; Lejzerowicz et al. 2015). Despite its enormous potential, metabarcoding is also subject to some biases. Understanding how various metabarcoding methodologies affect estimates of species richness ( $\alpha$ diversity) and patterns of community composition ( $\beta$ diversity) has therefore been an active area of research (Bik et al. 2012; Leray \& Knowlton 2016). For example, various empirical studies have shown that sampling techniques (Hirai et al. 2015), sample storage and DNA extraction methods (Brannock \& Halanych 2015; Deiner et al. 2015) induce taxon-specific biases. Others have highlighted how additional biases occur during PCR amplification as a result of differential primer binding efficiency (Elbrecht \& Leese 2015). Informatics procedures for data quality control (Schloss et al. 2011; Rossberg et al. 2014), sequence clustering (Brown et al. 2015; Flynn et al. 2015) and taxonomically biased public repositories (e.g. GenBank) (Guillou et al. 2013; Leray \& Knowlton 2016) are now wellidentified sources of variation in taxon detection. Together, these empirical studies quantifying biases have highlighted the importance of implementing rigorous experimental designs and adhering to strict laboratory protocols. They have also helped develop statistical frameworks that take into account methodological artifacts for the inference of taxon richness, biomass and community dissimilarity from sequence data (Shelton et al. 2016). Despite significant progress, the state of knowledge remains limited. Here, we further help to understand how metabarcoding sequence data can be used to make ecological predictions by exploring two potentially important sources of biases: indexed PCR primers and random sampling during Illumina MiSeq sequencing.

It is now well accepted that some level of primer bias is inevitable with PCR-based approaches because no primer set is truly universal (Leray et al. 2013). Differences in primer affinity among taxonomic groups prevent the detection of some OTUs and result in inaccurate estimates of relative abundances, particularly within complex samples (Pinto \& Raskin 2012; Deagle et al. 2013; Elbrecht \& Leese 2015; Piñol et al. 2015). Pervasive amplification biases of some primer sets have even led some to suggest that metabarcoding should only be interpreted in 
77 terms of OTU presence-absence, not in terms of relative abundance (Elbrecht \& Leese 2015;

78 Piñol et al. 2015). The addition of short indices to PCR primers has been suggested to induce

79 further amplification biases. Indexed PCR primers are commonly used to minimize the per

80 sample cost of sequencing by allowing numerous samples to be sequenced on a single run

81 (Binladen et al. 2007). Unique indices are attached to the 5' end of the forward or reverse primer

82 (or both) during oligonucleotide synthesis. Each sample is then amplified with a unique set of

83 indexed primers to add a "DNA identifier" to each amplified fragment, allowing reads produced

84 by parallel sequencing to be assigned to their original sample. This approach assumes that

85 indices differing by two to three base pairs do not modify the affinity of primers with DNA

86 templates during PCR cycles, an assumption that could be violated (Berry et al. 2011; O'Donnell

87 et al. 2016), particularly for highly diverse communities.

88 Another much more neglected source of variation in OTU detection may be attributed

89 solely to random sampling effects that are completely independent of any technical causes.

90 Random sampling, or the random draw of OTUs from a pool where OTUs have equal chances of

91 being selected, is most likely to occur during PCR amplification, library preparation (e.g. ligation

92 of adaptors) and sequencing (regardless of the platform used), with the greatest impact on the

93 detection of rare OTUs (Zhan \& MacIsaac 2015). To date, two studies that assessed the

94 reproducibility of amplicon-based studies proposed random sampling artifacts as a significant

95 concern for the reproducibility of presence-absence data. The first suggested that random

96 sampling was the main cause for the low levels of overlap in OTU composition between PCR

97 replicates of microbial communities (Zhou et al. 2011), but the authors used different indexed

98 PCR primers across technical replicates, a potential confounding source of bias. The second and

99 more recent study came to the same conclusion for metazoan communities, but they compared

100 biological replicates (i.e. two parallel fractions of the same sample) rather than technical

101 replicates (Zhan et al. 2014).

102 The present study was designed to explore the significance of sequence abundance

103 patterns and to disentangle technical biases caused by the addition of short indices to PCR

104 primers versus random sampling occurring during Illumina MiSeq sequencing. We analysed the

105 reproducibility of Illumina MiSeq data for a single mock sample comprising DNA of 34 marine

106 invertebrates belonging to six phyla. We targeted a short fragment of the mitochondrial

107 Cytochrome c Oxidase Subunit I (COI) with seven indexed broad-range primers. The COI gene, 
108

109

110

111

112

113

114

115

116

117 Preparation of the mock sample

118 Communities of small invertebrates were collected using Autonomous Reef Monitoring

119 Structures (ARMS) in the Indian River Lagoon of Florida following a standard protocol

120

121

122

123

124

125

126

127

128

129

130

131

132

133

134

135

136 Reference barcode library

137 Individual DNA extracts were used for PCR amplification and Sanger sequencing of a $\sim 658 \mathrm{bp}$

138 portion of the COI gene as described previously (Leray \& Knowlton 2015). Sequences obtained 
139 in both directions were assembled, checked for stop codons and frameshifts, and aligned in

140 Geneious (Biomatters) to build a reference library containing COI barcodes for each of the 34

141 specimens included in the mock sample. The complete list of specimens, including taxonomy

142 and GenBank accession numbers, are provided in Table S1. A PCR reaction performed with the

143 negative control extraction confirmed the absence of contaminants (no band on 1.5\% agarose

144 gel).

145

146 Illumina metabarcoding of the mock sample

147 Pooled genomic DNA (the mock sample) was used to amplify a $\sim 313 \mathrm{bp}$ of the COI region with

148 seven sets of indexed PCR primers [index-mlCOIintF/jgHCO-index (Leray et al. 2013)]. The

149 same index was used on the forward and reverse primer within each individual PCR

150 amplification (e.g. Primer set 1: Index1-mlCOIF/jgHCO-index 1; Primer set 2: Index2-

$151 \mathrm{mlCOIF} / \mathrm{jgHCO}$-index2) (Fig. 1, Table 1) to minimize the likelihood of false read-to-sample

152 assignments due to tag jumping (Schnell et al. 2015). Three PCR reactions (i.e. triplicates) were

153 performed using each of the seven indexed primer pairs for a total of 21 PCRs using the PCR

154 mixtures and touchdown temperature profile described previously (Leray \& Knowlton 2015).

155 PCR reactions were prepared in a room free of PCR amplicons. Triplicate PCRs were pooled (for

156 a total of seven pools) to limit stochastic amplification biases, and purified using Agencourt

157 AMPure XP beads. Cleaned PCR products were quantified with a Qubit fluorometer, and

158 equimolar amounts were combined into a single tube prior to library preparation using an adaptor

159 ligation approach (Leray et al. 2016). A total of $1 \mu \mathrm{g}$ of pooled amplicon in a final volume of

$16060 \mu 1$ was used for end repair, A-tailing and adaptor ligation following protocols and reagents of

161 the TruSeq PCR-free kit (Illumina). The addition of Illumina indexed-adaptors via ligation

162 prevents tag jumping caused by the formation of chimeric sequences during the bulk

163 amplification of tagged amplicons (cf. dual-PCR approach, Bourlat et al. 2016). The library was

164 validated via qPCR using the KAPA library quantification kit and diluted to a final concentration

165 of $4 \mathrm{nM}$. Paired-end sequencing of the library was performed twice on two separate runs of the

166 Illumina MiSeq platform at the Laboratory of Analytical Biology of the Smithsonian National

167 Museum of Natural History (Washington, DC, USA) using the Miseq reagent kit v3 (600

168 cycles). The same MiSeq instrument was used for both runs conducted one week apart from each 
169 other. Note that the library of the present study was combined in each MiSeq run with several

170 other unrelated libraries prepared with different TruSeq adaptors.

171

172 Analysis of the Illumina Miseq data

173 The script used to analyse the dataset is provided in Supporting file TextS1. Illumina forward

174 and reverse reads were denoised using BFC ( $\mathrm{Li} 2015)$, an error correcting tool designed

175 specifically for Illumina short reads. Denoised paired reads were merged into contigs in Usearch

1768.1 (Edgar 2010) allowing for a maximum of five mismatches and a maximum of zero gaps in

177 the overlapping region. Pairs were also discarded if the number of expected errors predicted by

178 Phred scores after merging (fastq_merge_maxee) was higher than one (Edgar \& Flyvbjerg 2015).

179 The fasta file was then demultiplexed based on the primer index in Mothur (Schloss et al. 2009)

180 and additional reads were discarded if they (1) had any mismatch in the primer and index region,

181 (2) did not have the same index on the forward and reverse primer to prevent false assignments

182 of reads due to tag jumping (Schnell et al. 2015), (3) had at least one homopolymer region longer 183 than 8bp, or (4) had any ambiguous base calls.

184 Sequences of both MiSeq runs were pooled, and the dataset was dereplicated in Mothur.

185 The option "enrichAlignment" implemented in MACSE (Ranwez et al. 2011) was then used to

186 align unique reads to the reference COI database of the Moorea Biocode project (7,675

187 sequences from 30 phyla represented) based on amino acid translations. We selected the

188 invertebrate mitochondrial translation code to perform the alignment and detect interruptions in

189 the open reading frame due to nucleotide substitution or nucleotide insertion/deletion. We only

190 retained sequences without any stop codons or frameshifts for subsequent analysis. To further

191 reduce the variability in the dataset, we used the preclustering approach implemented in Mothur

192 to merge reads differing by three or fewer than three bases. This algorithm ranks sequences in

193 order of their abundance and merges rare sequences with more abundant sequences within the

194 threshold specified. Reads were then screened for chimeras using UCHIME (Edgar et al. 2011),

195 and all remaining preclusters represented by a single sequence (singletons) were discarded.

196 High quality reads were clustered in Operational Taxonomic Units (OTUs) using CROP

197 (Hao et al. 2011), a Bayesian model that delineates OTUs based on the natural distribution of

198 sequence dissimilarity of the dataset. Rather than using a hard cut-off (e.g. 5\%), CROP generates

199 clusters within user-defined lower (-1) and upper $(-\mathrm{u})$ bound levels of similarity to account for 
200

201

202

203

204

205

206

207

208

209

210

211

212

213

214

215

216

217

218

219

220

221

222

223

224

225

226

227

228

229

230

differences in rates of sequence evolution among taxonomic groups. Here, we defined -13 and $-\mathrm{u}$ 4 because it was shown to create OTUs that closely reflect morphological species grouping among marine invertebrates by providing the lowest frequency of false positives (splitting of taxa) and false negatives (lumping of taxa) (Leray et al. 2013).

CROP outputs a file with one representative sequence per OTU that we used for taxonomic assignments. First, we ran local BLASTn searches in Geneious (Biomatters) to compare each representative sequence to the reference database containing COI barcodes of the 34 specimens included in the mock sample. Second, we conducted BLASTn searches against the full GenBank database and used the BOLD (Barcode of Life Data systems) search engine (Ratnasingham \& Hebert 2007) against all barcode records to identify OTUs that did not match a specimen included in the mock sample. Third, whenever sequence similarity to a reference barcode was lower than 97\%, we used a Bayesian phylogenetic approach implemented in the Statistical Assignment Package (SAP) to assign OTU representative sequences to a higher taxonomic group (see Leray et al. 2015 for further details).

Representative COI sequences (313bp) were used to infer phylogenetic relationships between OTUs using a Maximum Likelihood approach. We selected the best tree of 1000 maximum likelihood search replicates computed using the adaptive best tree search analyses implemented in GARLI v2.1 (Zwickl 2006) through the GARLI web service (Bazinet et al. 2014). We used a general time reversible nucleotide model with a proportion of invariant sites and among site rate heterogeneity modeled with a discrete gamma distribution $(\mathrm{GTR}+\mathrm{I}+\mathrm{G})$. GARLI default settings were used, including stepwise-addition starting trees.

\section{Analysis of dissimilarity in OTU composition}

Abundance data and taxonomic information for each OTU were summarized in a .biom formatted OTU table and imported into QIIME (Caporaso et al. 2010). To evaluate compositional dissimilarities among replicates (i.e. $\beta$ diversity) the OTU table was used to calculate distance matrices based on the Jaccard and the Bray Curtis metrics in Qiime (beta_diversity.py with -m binary_jaccard, bray_curtis). Note that the term "pseudo- $\beta$ " diversity may be more appropriate for describing dissimilarities in composition caused by methodological artifacts because they artificially inflate diversity (e.g. incomplete sampling, Manter \& Bakker 2015). However, we use the term $\beta$ diversity throughout for simplification. Jaccard considers 
231 only the presence-absence of OTUs; a value of 0 indicates that samples have exactly the same

232 OTU composition whereas a value of 1 indicates that samples do not have any OTUs in

233 common. Because indexed primers and random sampling during sequencing are more likely to

234 affect the repeatability of rare OTUs, we repeated calculations of pairwise distances after

235 sequentially discarding rare OTUs from the OTU table. In brief, we repeated calculations of

236 Jaccard after removing OTUs represented by less than three sequences from the dataset, then

237 after removing OTUs represented by four sequences and so on, until a dataset from which all

238 OTUs represented by less than 30 sequences were removed. On the other hand, Bray-Curtis takes

239 into account differences in abundance of reads between samples; a value of 0 indicates that

240 samples are exactly identical in terms of OTU composition and abundance of reads whereas a

241 value of 1 indicates that samples do not have any OTUs in common. It is much less sensitive to

242 rare OTUs, resulting in low distance values when communities have shared abundant OTUs.

243 We assessed bias by calculating the pairwise Jaccard and Bray-Curtis at two levels of

244 replication: primer index replicate and sequencing replicate. Within-primers pairwise

245 dissimilarities were calculated between communities obtained using identical primer indices but

246 sequenced in different MiSeq runs (i.e. primer1/run1 vs. primer1/run2; primer2/run1 vs.

247 primer2/run2). Between-primers pairwise dissimilarities were calculated between communities

248 obtained using different primer indices regardless of the sequencing run (i.e. primer1/run1 vs.

249 primer2/run1; primer2/run1 vs. primer1/run2).

250 Within-run pairwise dissimilarities were calculated between communities obtained within

251 the same MiSeq sequencing run regardless of the indexed primer set used (i.e. primer1/run1 vs.

252 primer2/run1; primer2/run1 vs. primer3/run1). Between-run pairwise dissimilarities were

253 calculated between communities obtained in different MiSeq sequencing runs regardless of the

254 indexed primer set used (i.e. primer1/run1 vs. primer1/run2; primer1/run1 vs. primer2/run2).

255 Pairwise community dissimilarities calculated within any indexed primer result from discordance

256 that occurred after library preparation. Dissimilarities calculated between and within MiSeq runs

257 as well as between indexed primers within a run result from a combination of technical and

258 random sampling artifacts occurring during PCR amplification, library preparation and

259 sequencing (Table 2). To further examine similarities in OTU composition, we calculated

260 hierarchical cluster trees using an Unweighted Pair Group Method with Arithmetic mean

261 (UPGMA) based on Jaccard and Bray-Curtis. Branch support was calculated by jackknifing the 
262 dataset 100 times using $75 \%$ of the sequences in the smallest sample $(34,206)$. We also

263 visualized Bray-Curtis differences between samples using a principal coordinate analyses

264 (PCoA). The score of each OTU was plotted in 2-dimensional PCoA space to illustrate their

265 influence on the dissimilarities between samples. Finally, we tested differences in OTU

266 composition between primer indices and sequencing runs using permutational multivariate

267 analysis (PERMANOVA, Anderson 2001) computed using 10,000 permutations within the R

268 package Vegan (Oksanen et al. 2009). Because differences in sequencing depth can affect

269 estimates of $\alpha$ and $\beta$ diversity, all analyses were repeated with a dataset rarefied down to the

270 lowest number of sequences that a sample contained $(45,609)$.

271

272 RESULTS

273

274 Summary of sequencing runs

275 Illumina MiSeq sequencing runs provided a total of 779,758 (run 1) and 745,490 (run 2) raw

276 paired end reads, of which 580,938 (74.5\%) and 562,507 (75.4\%) were successfully merged into

277 contigs. Most paired reads that failed to merge (95.5\% and $95.7 \%$, respectively) had a number of

278 expected errors above one. A total of 80,035 and 94,590 additional reads were discarded because

279 they had at least one mismatch in the primer or index region, had a different index on the

280 forward and reverse primer, had at least one homopolymer region longer than 8bp, or had one or

281 more ambiguous base calls. Out of the 500,903 (64.2\%) and 467,917 (62.7\%) remaining reads,

28223,655 and 22,173 had at least one frameshift or stop codon. In total, we obtained a dataset with

283 469,352 (60.2\%) and 438,752 (58.8\%) high quality paired reads in Miseq run 1 and 2,

284 respectively. Within each Miseq run, the number of reads per indexed PCR ranged from 50,150

285 to 75,916 (mean $\pm \mathrm{SD}=67,030 \pm 12,010$ ) and from 45,609 to 78,387 (mean $\pm \mathrm{SD}=62,664$

$286 \pm 11,885)$, respectively. The raw Illumina MiSeq and the final dataset are available from Figshare

287 (MiSeq Run1, R1 direction:

288 https://dx.doi.org/10.6084/m9.figshare.4039821.v1; MiSeq Run1, R2 direction:

289 https://dx.doi.org/10.6084/m9.figshare.4039860.v1; MiSeq Run2, R1 direction:

290 https://dx.doi.org/10.6084/m9.figshare.4039893.v1; MiSeq Run2, R2 direction:

291 https://dx.doi.org/10.6084/m9.figshare.4039899.v1)

292 
293 Diversity and abundance

294 The Bayesian clustering tool CROP delineated 128 Operational Taxonomic Units (OTUs). Six

295 bacterial OTUs and two OTUs matching contaminants (Homo sapiens and a rodent) representing 296 a total of 244 sequences were removed from the dataset, leaving a total of 120 eukaryotic OTUs.

297 BLASTn searches against the reference barcode library revealed that 34 of these OTUs 298 corresponded to the 34 species included in the mock sample. We will refer to these OTUs as 299 "target OTUs". The 86 OTUs that did not match any species included in the mock sample are 300 hereafter referred to as "non-target OTUs". Among them, $31(25.8 \%)$ had $>97 \%$ similarity to 301 GenBank and BOLD sequences while 41 (34.2\%) could be confidently assigned to higher 302 taxonomic levels using the Bayesian phylogenetic approach implemented in SAP, leaving only 30314 OTUs (11.7\%) unidentified. As noted above, the 34 target OTUs belonged to six animal phyla 304 (Table S1) whereas the 72 identified non-target OTUs were more diverse, with representatives of 30512 phyla (Fig. 2, Fig. S1). Among them, Arthropoda (23 OTUs), Annelida (11 OTUs), 306 Rhodophyta (8 OTUs) and Porifera (7 OTUs) were the most common. Other photosynthetic 307 organisms included three diatoms (phylum Bacillariophyta) and two brown algae (phylum 308 Ochrophyta).

Among non-target OTUs, 20 corresponded to animals that typically live attached to a 310 hard substrate (e.g. Hydrozoa, Ascidiacea). Moreover, one OTU belonged to a group of parasitic 311 copepods known to live on fish hosts (Siphonostomatoida). A total of 44 additional OTUs (51\%) 312 matched species or OTUs previously reported in the Indian River Lagoon of Florida [(Leray \& 313 Knowlton 2015), http://www.sms.si.edu/IRLspec/].

314 The rank abundance curve showed relatively few common OTUs followed by a long tail 315 of rare taxa (Fig. 3). The 34 target OTUs were by far the most represented, accounting for $99.7 \%$ 316 of the reads in each of two Miseq runs. In contrast, the 86 non-target OTUs accounted for only $3170.3 \%$ of reads (1351 and 1289 reads in each run). Only one of these had more sequence reads 318 than some of the target OTUs; it accounted for 1162 and 1018 reads in Miseq run 1 and 2, 319 respectively, but was unidentified. Most of the non-target OTUs were very rare in the dataset (79 320 of 86 with $<10$ sequences).

321 Despite pooling equimolar amounts of total genomic DNA, the total number of reads for 322 each target OTU spanned three orders of magnitude. Based on expected number of reads 323 calculated using the total amount of genomic DNA, 8 OTUs were highly under-represented 
$324(<4,000$ sequences), while two taxa were highly over-represented ( $>80,000$ sequences) (Fig. 4A).

325 At the phylum level, the bias was less pronounced, but observed proportions of reads also

326 differed from what was expected based on ratios of total genomic DNA pooled (Fig. 4B).

327 Arthropoda (rank 1, Fig. 4B) and Platyhelminthes (rank 6, Fig. 4B) were underrepresented while

328 Annelida (rank 2, Fig. 4B) was over represented.

329 The 34 target OTUs were present in every one of the seven different indexed PCR trials

330 (mean $\pm \mathrm{SD}=34 \pm 0.0$ ). On the other hand, the 86 non-target OTUs appeared much less

331 consistently; the mean $( \pm \mathrm{SD})$ number of non-target OTUs per indexed PCR trial was $16( \pm 4.4)$

332 and $21( \pm 3.7)$ in MiSeq runs 1 and 2, respectively. After rarefying the dataset down to the lowest

333 number of reads $(45,609)$ to account for differences in sequencing depth, all target OTUs were

334 still detected in all seven indexed PCR trials, whereas the mean $( \pm \mathrm{SD})$ number of non-target

335 OTUs per indexed PCR trial was only $12.6( \pm 3.9)$ and $18.6( \pm 2.9)$ in the two sequencing runs.

336 The mean total number of OTUs was significantly different between MiSeq runs based on both

337 the non-rarefied $(t=-2.28, \mathrm{df}=11.6, P=0.04)$ and the rarefied $(t=-3.27, \mathrm{df}=11.2, P=0.01)$

338 datasets.

339

340 Dissimilarity in OTU composition

341 A total of 40 of the 86 non-target OTUs (46\%) were only present in one of the two MiSeq runs, a

342 majority of which (26) were represented by only two sequences. Rarefying the OTU table

343 yielded similar results (e.g. 44 OTUs were present in a single MiSeq run). We repeated the

344 rarefaction draws five times and found no differences in the general pattern as a result of random

345 subsampling (Fig. S2). We present the results obtained using one of the rarefied datasets (draw 1

346 in Fig. S2) below.

$347 \quad$ Pairwise $\beta$ Jaccard dissimilarities (compositional similarities among replicates in terms of 348 presence-absence) ranged from 0.24 to 0.43 between runs and from 0.18 to 0.45 within a run (see

349 Table 2 for a summary of pairwise dissimilarity calculations). Values of $\beta$ Jaccard also ranged

350 from 0.28 and 0.39 within indexed primers across sequencing runs because of the presence of 351 rare OTUs.

352 There was a relationship between the presence of non-target OTUs in indexed PCR trials

353 and the number of reads they represented in the dataset (Fig. 5). As a result, values of $\beta$ Jaccard

354 dissimilarities gradually decreased with the removal of rare OTUs (Fig. 6). Pairwise values 
355 calculated within and between runs were close to 0 after all OTUs represented by less than eight 356 sequences were removed (Fig. 6A and 6B). The same pattern was observed within and between 357 indexed primers (Fig. 6C and 6D). All values of $\beta$ Jaccard dissimilarities were equal to 0

358 (composition exactly the same) after OTUs with less than 30 sequences were removed from the 359 dataset.

360

361

362

363 primers.

364 communities) based on the Jaccard (presence-absence) analysis showed that there was no 366 significant grouping among indexed PCR trials or among Illumina runs (Fig. 8A). Indexed PCRs explained a higher fraction of the variance in Jaccard dissimilarity $\left(\mathrm{R}^{2}=47.4 \%\right)$ than Illumina runs $\left(\mathrm{R}^{2}=7.8 \%\right)$ in PERMANOVA analysis (Table 3$)$, but there were no significant differences between indexed PCRs $\left(\mathrm{F}_{(1,6)}=1.059, p=0.367\right)$ or between Illumina runs $\left(\mathrm{F}_{(1,6)}=1.049, p=\right.$ 0.414). Calculations based on an OTU table from which the rare OTUs ( $<8$ sequences) were removed showed a similar lack of structure (Fig. 8B). On the other hand, the Bray-Curtis UPGMA tree displayed a consistent clustering of indexed PCR trials obtained from the two Ilumina MiSeq runs (Fig. 8C). PERMANOVA analysis confirmed that much of the variation in read abundance was driven by indexed PCRs $\left(\mathrm{R}^{2}=98.3 \%, \mathrm{~F}_{(1,6)}=1.059, p=0.367\right)$ but differences were also significant between Illumina runs $\left(\mathrm{R}^{2}=0.7 \%, \mathrm{~F}_{(1,6)}=1.049, p=0.414\right)$. Indexed primer sets 2 and 3 appeared as outliers on the UPGMA tree (Fig. 8C) and PCoA plot (Fig. 9). OTUs 11 (Cilicaea) and 34 (Polychaeta) drove differences between primer set 2 and the rest of the PCR trials while OTUs 80 (Hypleurochilus geminatus), 123 (Menippe mercenaria) and 114 (Polychaeta) played a significant role at differentiating primer set 3 from the rest of the 380 dataset.

\section{DISCUSSION}

Before metabarcoding can be routine in biodiversity monitoring, it is essential to quantify all 385 potential sources of variability in diversity estimates. Our study aimed to evaluate the effect of 
386 387

388

389

390

391

392

393

394

395

396

397

398

399

400

401

402

403

404

405

406

407

408

409

410

411

412

413

414

415

416

indexed PCRs and random sampling in MiSeq sequencing on the reproducibility of metabarcoding.

We recovered a much higher total number of OTUs (120) than expected based on the known composition of the mock sample of 34 organisms. However, most of these non-target sequences could be identified, and we used stringent data acceptance protocols, suggesting that most of these sequences represented genuine but rare OTUs (i.e., taxa or products of taxa that were present in trace amounts in the original mock sample but undetected when it was created) rather than DNA artifacts. Most non-target OTUs were represented by very few sequences (with one notable exception), but the relative abundance of target OTUs varied by up to three orders of magnitude.

By comparing results between different index PCR trials and sequencing runs, we show that the noise in presence-absence data between replicates is most likely the result of random sampling of rare taxa, which causes the low reproducibility of rare OTUs. On the other hand, we found that indexed PCRs accounted for a larger amount of variation in read numbers than random sampling during sequencing.

Metabarcoding is known to be a much more sensitive measure of biodiversity than traditional morphological surveys because any trace of DNA in the sample is potentially detectable. As such, the presence of numerous OTUs (86) that did not match any of the 34 species comprising the mock sample is not surprising. Species level assignment of 31 OTUs to GenBank and BOLD reference barcodes ( $>97 \%$ similarity) confirms that they all belonged to the local Floridian fauna, not common laboratory contaminants. A total of 20 sessile and 13 protist taxa were likely present on the legs of spider crabs and other arthropods. In fact, epiphytes, endosymbionts and also parasites are commonly described in sequence libraries (Geisen et al. 2015; de Vargas et al. 2015). Motile OTUs (e.g. among arthropods, molluscs and echinoderms) may be part of the diet of carnivorous annelids and thus present in the section of their digestive tract that was used for DNA extraction. Sequences of non-target OTUs may also originate from eDNA traces (Kelly et al. 2014), such as excreted cells, faeces, epidermal tissue, hair and body fluids from dead individuals, especially given that many specimens were in close proximity in a tray for several hours during field collecting. Interestingly, 14 OTUs could not confidently be assigned to any taxonomic level using the Bayesian Phylogenetic approach implemented in SAP. According to their phylogenetic placement (Fig. 2, Fig. S1) and their most similar BLASTn hits 
417 in GenBank and BOLD (not shown), most of these OTUs likely belong to single-celled

418 eukaryotes that are highly underrepresented in public databases.

419 Alternatively, the presence of artifacts among unidentified non-target OTUs cannot be

420 excluded despite stringent molecular (e.g. negative DNA extractions and PCRs, separate pre- and 421 post-PCR areas) and data analysis (e.g. amino acid alignment) procedures. For example, spurious

422 OTUs that result from sequencing errors or PCR chimeras may still account for some of the non423 target OTUs (e.g. long branches, Fig. 2), but they most likely represent a minor fraction of the 424 reads. Non-functional copies of COI transposed to the nuclear genome (numts) may also inflate 425 diversity if they have not accumulated enough variation for interruptions in the open reading 426 frame to occur. Numts are relatively common among marine invertebrates (e.g. decapods, 427 Williams \& Knowlton 2001) but a majority include stop codons and frameshifts (Song et al. 428 2008) and have therefore been discarded. PCR and sequencing of COI transcripts obtained from 429 the reverse transcription of cDNA from mRNA would ultimately ensure the removal of all non430 functional copies (Williams \& Knowlton 2001; Machida et al. 2009). Contaminations across 431 libraries during the sequencing reaction are another potential source of foreign DNA. This occurs 432 when mixed clusters form on the Illumina flowcell during bridge amplification. This 433 phenomenon may result in reads that have a different Illumina index than the one they were 434 assigned (cf. bleeding effect; Kircher et al. 2012, Schnell et al. 2015). We used local BLASTn 435 searches to compare OTUs detected in the present study to sequences from libraries (independent 436 studies) sequenced simultaneously in the MiSeq runs. We found that only eight rare OTUs had a $437>97 \%$ similarity between the two datasets which confirms previous findings (Schnell et al. 2015) 438 that cross-library contamination is not a major source of error.

439 Our results confirm the existence of an amplification bias between taxa. While most 440 target OTUs were represented by between $\sim 10,000$ and $\sim 60,000$ sequences, a few were either 441 highly over- or under-represented. As observed previously (Leray \& Knowlton 2015), effects of 442 primer biases tend to decrease as we group OTUs in higher taxonomic levels (Fig. 4B) which 443 suggests that the number of reads could be used as proxy for biomass of functional groups. 444 Importantly, some biological factors may also affect quantitative estimates. For example, 445 amounts of mitochondrial DNA differ between and even within tissue types as a function of 446 energetic demand (Fernandez-Vizarra et al. 2011). Therefore, our subsampling strategy may also 447 account for some of the variation observed. 
448

449

450

451

452

453

454

455

456

457

458

459

460

461

462

463

464

465

466

467

468

469

470

471

472

473

474

475

476

477

478

Despite its increasing use for biodiversity monitoring, the reproducibility of metabarcoding datasets has only rarely been evaluated. Our study demonstrates that the reproducibility of OTUs can be predicted from the total number of reads they represent in the dataset. Abundant OTUs are highly reproducible, whereas low abundance (rare) OTUs were never consistently found across PCR replicates and sequencing runs. These findings have important implications for estimates of $\beta$ diversity calculated based on presence-absence data because the inclusion of rare OTUs may induce a background noise (high $\beta$ diversity) that could mask real ecological patterns.

The high level of noise between the two replicate sequencing runs is one of the most surprising findings of this study. Because sequencing replicates were derived from an individual library, the discrepancy in presence-absence data must have occurred during the generation of the templates on the flow cell. McIntyre and colleagues (2011) reported similar inconsistencies in the detection of low-abundance transcripts and proposed that the observed level of noise was expected under a scenario of random variation. They calculated that less than $0.002 \%$ of the total given molecules loaded on an Illumina platform were effectively sequenced because a very small portion binds to the flow cell.

Our initial assumption was that randomness would account for a minor level of variation in OTU presence-absence in comparison to the noise caused by indexed PCR primers. This is because previous studies have shown that small tags could affect the binding efficiency of primers across taxa (O’Donnell et al. 2016). However, our results do not support this. First, the effect of rare OTUs was nearly as pronounced on $\beta$ Jaccard values within indexed primer trials (Fig. 6C) (random sampling only), as it was between indexed primers as well as within and between sequencing runs (Fig. 6D, 6A and 6B respectively) (random sampling combined with potential technical artifacts). Second, despite accounting for the largest fraction of the variance in Jaccard dissimilarity (47.4\%, Table 3), differences between indexed PCRs were not significant in PERMANOVA analysis, and indexed primers did not cluster together on UPGMA trees [e.g. Indexed P1 from run 1 and 2 are not grouped together (Fig. 8A and 8B)]. This indicates that levels of technical biases due to primer indices are not a significant factor affecting the presenceabsence of OTUs. On the other hand, our results show that indexed PCRs induced a higher level of noise in relative abundance data than sequencing alone (Fig. 8C, Table 3). The lack of evidence for taxon-specific biases suggests that a combination of technical factors (e.g. minor 
479

480

481

482

483

484

485

486

487

488

489

490

491

492

493

494

495

496

497

498

499

500

501

502

503

504

505

506

507

508

509

differences in binding efficiency) and stochastic events during early PCR cycles could be responsible for this pattern. A double PCR approach, that consists of a first PCR of genomic DNA template with non-indexed primers, followed by a second PCR of the template of the first reaction with indexed primer (Bourlat et al. 2016), might help alleviate differences among replicates if the noise is caused by the indices (Berry et al. 2011; O’Donnell et al. 2016). Ultimately, bioinformatics advances should make it possible to use non-PCR based approaches on complex environmental samples of eukaryotes (Tang et al. 2015).

\section{CONCLUSION}

Our study demonstrates that the random sampling effect of the sequencing process causes differences among replicates, artificially inflating $\beta$-diversity. Its effect is particularly significant on presence-absence data that is deemed the most reliable product of metabarcoding experiments (as opposed to abundance data). On the other hand, we show that many of the less reproducible OTUs represent genuine taxa occurring in low abundance in the community. Therefore, the strategy for handling rare OTUs should vary according to the objectives of the study and how sensitive the interpretation of the data may be to the presence of false positives or false negatives.

In environmental biomonitoring studies that use common $\beta$ descriptors based on presence-absence, non-reproducible OTUs may obscure patterns of community turn-over through time or along spatial gradients. As a result, some authors have recommended the systematic removal of rare OTUs such as doubletons and tripletons (Kunin et al. 2010). Here, our dataset shows that the cut-off for reproducibility across all replicates is much higher than previously suggested. $\beta$ Jaccard diversity reached values close to 0 only if OTUs containing less than eight sequences $(0.02 \%$ of reads $)$ were removed, which comes at the price of discarding valuable ecological data (e.g. true detection of epiphytes). However, note that alternative $\beta$ diversity metrics that give less weight to rare taxa will be less sensitive to undersampling (Beck et al. 2013) and consequently, allow more relaxed cut-offs.

The systematic exclusion of rare OTUs, under the assumption that they are most likely false positive records, will be particularly detrimental to studies seeking to investigate the distribution of specific taxa. For example, non-indigenous species present in the form of 
510 propagules in the environment may not be consistently detected across replicates because of their

511 low relative abundance (Zaiko et al. 2015). Similarly, such an approach may underestimate the

512 contribution of highly digested and small prey items in food webs (Leray et al. 2015). Rather

513 than systematically removing OTUs based on arbitrary detection cut-offs, some have therefore

514 advocated coupling metabarcoding data with statistical methods that account for the presence of

515 false positives (Lahoz-Monfort et al. 2016). Given replicate PCR assays or replicate samples

516 within sites, occupancy models infer the probability of detection of individual species given the

517 rate of imperfect detection (i.e. false positives). For example, this approach was successfully

518 used to estimate the probability of occurrence of the invasive Burmese python in South Florida

519 (Hunter et al. 2015) with important conservation implications. When possible, the simultaneous

520 collection of data less prone to false positives (e.g. visual surveys) may prove useful to

521 corroborate the presence of ambiguous detections (i.e. taxa that are not detected across all PCR

522 replicates) and estimate the probability of false negatives in site occupancy models (Chambert et

523 al. 2015; Miller et al. 2015).

524 Regardless of the application, our results further reinforce the importance of the inclusion

525 of technical replicates (parallel PCR and sequencing from the same sample) in metabarcoding

526 experimental designs. Data reproducibility should be determined empirically as it will depend

527 upon the sequencing depth, the type of sample, the sequence analysis pipeline, and the number of 528 replicates.

529

\section{ACKNOWLEDGEMENTS}

531 We thank Amanda Devine and Jeffrey Hunt for logistical support as well as Matthew Kweskin

532 and Paul Frandsen for informatics support. James Lawrence O’Donnell and Emmett Duffy

533 provided constructive comments on an earlier version of the manuscript. We are grateful to Ryan

534 Kelly and Rupert Collins for their helpful suggestions that improved the clarity of the manuscript

535 as well as Tomas Hrbek for editing the paper. This is contribution \#13 from the Smithsonian's

536 MarineGEO Network and contribution \#1049 from the Smithsonian Marine Station at Fort

537 Pierce.

538

539

540

REFERENCES 
541 Anderson, M.J. (2001). A new method for non-parametric multivariate analysis of variance.

542 Austral Ecology, 26, 32-46.

543 Bazinet, A.L., Zwickl, D.J. \& Cummings, M.P. (2014). A gateway for phylogenetic analysis

544 powered by grid computing featuring GARLI 2.0. Systematic Biology, 63, 812-8.

545 Beck, J., Holloway, J.D. \& Schwanghart, W. (2013). Undersampling and the measurement of

546 beta diversity. Methods in Ecology and Evolution, 4, 370-382.

547 Berry, D., Ben Mahfoudh, K., Wagner, M. \& Loy, A. (2011). Barcoded primers used in

548 multiplex amplicon pyrosequencing bias amplification. Applied and Environmental

549 Microbiology, 77, 7846-9.

550 Bik, H.M., Porazinska, D.L., Creer, S., Caporaso, J.G., Knight, R. \& Thomas, W.K. (2012).

551 Sequencing our way towards understanding global eukaryotic biodiversity. Trends in 552 Ecology \& Evolution, 27, 233-243.

553 Binladen, J., Gilbert, M.T.P., Bollback, J.P., Panitz, F., Bendixen, C., Nielsen, R. \& Willerslev,

554

555

556

557

558

559

560

561

562

563

564

565

566

567

568

569

570

571 E. (2007). The use of coded PCR primers enables high-throughput sequencing of multiple homolog amplification products by 454 parallel sequencing. Plos One, 2, e197.

Bourlat, S.J., Haenel, Q., Finnman, J. \& Leray, M. (2016). Preparation of amplicon libraries for metabarcoding of marine eukaryotes using Illumina MiSeq: The dual-PCR method. Methods in Molecular Biology: Marine Genomics Methods and Protocols (ed S.J. Bourlat), pp. 197-207. Springer Protocols.

Brannock, P.M. \& Halanych, K.M. (2015). Meiofaunal community analysis by high-throughput sequencing: comparison of extraction, quality filtering, and clustering methods. Marine Genomics, 23, 67-75.

Brown, E.A., Chain, F.J.J., Crease, T.J., MacIsaac, H.J. \& Cristescu, M.E. (2015). Divergence thresholds and divergent biodiversity estimates: can metabarcoding reliably describe zooplankton communities? Ecology and Evolution, 5, 2234- 2251.

Caporaso, J.G., Kuczynski, J., Stombaugh, J., Bittinger, K., Bushman, F.D., Costello, E.K., Fierer, N., Peña, A.G., Goodrich, J.K., Gordon, J.I., Huttley, G.A., Kelley, S.T., Knights, D., Koenig, J.E., Ley, R.E., Lozupone, C.A., McDonald, D., Muegge, B.D., Pirrung, M., Reeder, J., Sevinsky, J.R., Turnbaugh, P.J., Walters, W.A., Widmann, J., Yatsunenko, T., Zaneveld, J. \& Knight, R. (2010). QIIME allows analysis of high-throughput community sequencing data. Nature Methods, 7, 335-6. 
572 Chambert, T.C., Miller, D.A.W. \& Nichols, J.D. (2015). Modeling false positive detections in 573 species occurrence data under different study designs. Ecology, 96, 332-339.

574 Deagle, B.E., Thomas, A.C., Shaffer, A.K., Trites, A.W. \& Jarman, S.N. (2013). Quantifying 575 sequence proportions in a DNA-based diet study using Ion Torrent amplicon sequencing:

576

577

578

579

580

581

582

583

584

585

586

587

588

589

590

591

592

593

594

595

596

597

598

599

600

601

602 which counts count? Molecular Ecology Resources, 13, 620-33.

Deiner, K., Walser, J.-C., Mächler, E. \& Altermatt, F. (2015). Choice of capture and extraction methods affect detection of freshwater biodiversity from environmental DNA. Biological Conservation, 183, 53-63.

Edgar, R.C. (2010). Search and clustering orders of magnitude faster than BLAST. Bioinformatics, 26, 2460-1.

Edgar, R.C. \& Flyvbjerg, H. (2015). Error filtering, pair assembly, and error correction for nextgeneration sequencing reads. Bioinformatics, 31, 3476-3482.

Edgar, R.C., Haas, B.J., Clemente, J.C., Quince, C. \& Knight, R. (2011). UCHIME improves sensitivity and speed of chimera detection. Bioinformatics, 27, 2194-2200.

Elbrecht, V. \& Leese, F. (2015). Can DNA-based ecosystem assessments quantify species abundance? Testing primer bias and biomass - sequence relationships with an innovative metabarcoding protocol. Plos One, 10, e0130324.

Fernandez-Vizarra, E., Enriquez, J.A., Perez-Martos, A., Montoya, J. \& Fernandez-Silva, P. (2011). Tissue-specific differences in mitochondrial activity and biogenesis. Mitochondrion, 11, 207-213.

Flynn, J.M., Brown, E.A., Chain, F.J.J., MacIsaac, H.J. \& Cristescu, M.E. (2015). Toward accurate molecular identification of species in complex environmental samples: testing the performance of sequence filtering and clustering methods. Ecology and Evolution, 5, 22522266.

Geisen, S., Laros, I., Vizcaíno, A., Bonkowski, M. \& de Groot, G.A. (2015). Not all are freeliving: high-throughput DNA metabarcoding reveals a diverse community of protists parasitizing soil metazoa. Molecular Ecology, 24, 4556-4569.

Guillou, L., Bachar, D., Audic, S., Bass, D., Berney, C., Bittner, L., Boutte, C., Burgaud, G., de Vargas, C., Decelle, J., Del Campo, J., Dolan, J.R., Dunthorn, M., Edvardsen, B., Holzmann, M., Kooistra, W.H.C.F., Lara, E., Le Bescot, N., Logares, R., Mahé, F., Massana, R., Montresor, M., Morard, R., Not, F., Pawlowski, J., Probert, I., Sauvadet, A.- 
603

604

605

606

607

608

609

610

611

612

613

614

615

616

617

618

619

620

621

622

623

624

625

626

627

628

629

630

631

632

633

L., Siano, R., Stoeck, T., Vaulot, D., Zimmermann, P. \& Christen, R. (2013). The Protist Ribosomal Reference database (PR2): a catalog of unicellular eukaryote small sub-unit rRNA sequences with curated taxonomy. Nucleic Acids Research, 41, D597-604.

Hao, X., Jiang, R. \& Chen, T. (2011). Clustering 16S rRNA for OTU prediction: a method of unsupervised Bayesian clustering. Bioinformatics, 27, 611-8.

Hirai, J., Yasuike, M., Fujiwara, A., Nakamura, Y., Hamaoka, S., Katakura, S., Takano, Y. \& Nagai, S. (2015). Effects of plankton net characteristics on metagenetic community analysis of metazoan zooplankton in a coastal marine ecosystem. Journal of Experimental Marine Biology and Ecology, 469, 36-43.

Hunter, M.E., Oyler-McCance, S.J., Dorazio, R.M., Fike, J.A., Smith, B.J., Hunter, C.T., Reed, R.N. \& Hart, K.M. (2015). Environmental DNA (eDNA) sampling improves occurrence and detection estimates of invasive Burmese pythons. PLOS ONE, 10, 1-17.

Ji, Y., Ashton, L., Pedley, S.M., Edwards, D.P., Tang, Y., Nakamura, A., Kitching, R., Dolman, P.M., Woodcock, P., Edwards, F. a., Larsen, T.H., Hsu, W.W., Benedick, S., Hamer, K.C., Wilcove, D.S., Bruce, C., Wang, X., Levi, T., Lott, M., Emerson, B.C. \& Yu, D.W. (2013). Reliable, verifiable and efficient monitoring of biodiversity via metabarcoding. Ecology Letters, 16, 1245-1257.

Kelly, R.P., Port, J.A., Yamahara, K.M. \& Crowder, L.B. (2014). Using environmental DNA to census marine fishes in a large mesocosm. PloS One, 9, e86175.

Kircher, M., Sawyer, S. \& Meyer, M. (2012). Double indexing overcomes inaccuracies in multiplex sequencing on the Illumina platform. Nucleic Acids Research, 40, e3-e3.

Kunin, V., Engelbrektson, A., Ochman, H. \& Hugenholtz, P. (2010). Wrinkles in the rare biosphere: pyrosequencing errors can lead to artificial inflation of diversity estimates. Environmental Microbiology, 12, 118-23.

Lahoz-Monfort, J.J., Guillera-Arroita, G. \& Tingley, R. (2016). Statistical approaches to account for false-positive errors in environmental DNA samples. Molecular Ecology Resources, 16, $673-685$.

Lejzerowicz, F., Esling, P., Pillet, L., Wilding, T.A., Black, K.D. \& Pawlowski, J. (2015). Highthroughput sequencing and morphology perform equally well for benthic monitoring of marine ecosystems. Scientific Reports, 5, 13932.

Leray, M., Haenel, Q. \& Bourlat, S.J. (2016). Preparation of amplicon libraries for 
634

635

636

637

638

639

640

641

642

643

644

645

646

647

648

649

650

651

652

653

654

655

656

657

658

659

660

661

662

663

664

metabarcoding of marine eukaryotes using Illumina MiSeq: The adapter ligation method. Methods in Molecular Biology: Marine Genomics Methods and Protocols (ed S.J. Bourlat), pp. 209-218. Springer Protocols.

Leray, M. \& Knowlton, N. (2016). Censusing marine eukaryotic diversity in the twenty-first century. Philosophical Transactions of the Royal Society of London. Series B, Biological Sciences, 371, 20150331.

Leray, M. \& Knowlton, N. (2015). DNA barcoding and metabarcoding of standardized samples reveal patterns of marine benthic diversity. Proceedings of the National Academy of Sciences of the United States of America, 112, 2076-2081.

Leray, M., Meyer, C.P. \& Mills, S.C. (2015). Metabarcoding dietary analysis of coral dwelling predatory fish demonstrates the minor contribution of coral mutualists to their highly partitioned, generalist diet. PeerJ, 3, e1047.

Leray, M., Yang, J.Y., Meyer, C.P., Mills, S.C., Agudelo, N., Ranwez, V., Boehm, J.T. \& Machida, R.J. (2013). A new versatile primer set targeting a short fragment of the mitochondrial COI region for metabarcoding metazoan diversity: application for characterizing coral reef fish gut contents. Frontiers in Zoology, 10, 34.

Li, H. (2015). BFC: correcting Illumina sequencing errors. Bioinformatics, 31, 2885-7.

Machida, R.J., Hashiguchi, Y., Nishida, M. \& Nishida, S. (2009). Zooplankton diversity analysis through single-gene sequencing of a community sample. BMC Genomics, 10, 438.

Manter, D.K. \& Bakker, M.G. (2015). Estimating beta diversity for under-sampled communities using the variably weighted Odum dissimilarity index and OTUshuff. Bioinformatics, 31, 3451-9.

McIntyre, L.M., Lopiano, K.K., Morse, A.M., Amin, V., Oberg, A.L., Young, L.J. \& Nuzhdin, S. V. (2011). RNA-seq: technical variability and sampling. BMC Genomics, 12, 293.

Miller, D.A.W., Bailey, L.L., Grant, E.H.C., Mcclintock, B.T., Weir, L.A. \& Simons, T.R. (2015). Performance of species occurrence estimators when basic assumptions are not met: a test using field data where true occupancy status is known. Methods in Ecology and Evolution, 6, 557-565.

O’Donnell, J.L., Kelly, R.P., Lowell, N.C. \& Port, J.A. (2016). Indexed PCR primers induce template-specific bias in large-scale DNA sequencing studies. PloS One, 11, e0148698. Oksanen, J., Kindt, R., Legendre, P., O’Hara, B., Simpson, G.L., Solymos, P., Stevens, M.H.H. 
665

666

667

668

669

670

671

672

673

674

675

676

677

678

679

680

681

682

683

684

685

686

687

688

689

690

691

692

693

694

695

\& Wagner, H. (2009). Vegan: community ecology package. R package version 1.15-3.

Piñol, J., Mir, G., Gomez-Polo, P. \& Agustí, N. (2015). Universal and blocking primer mismatches limit the use of high-throughput DNA sequencing for the quantitative metabarcoding of arthropods. Molecular Ecology Resources, 15, 819-30.

Pinto, A.J. \& Raskin, L. (2012). PCR biases distort bacterial and archaeal community structure in pyrosequencing datasets. PloS One, 7, e43093.

Ranwez, V., Harispe, S., Delsuc, F. \& Douzery, E.J.P. (2011). MACSE: Multiple Alignment of Coding SEquences accounting for frameshifts and stop codons. PloS One, 6, e22594.

Ratnasingham, S. \& Hebert, P.D.N. (2007). BOLD: The Barcode of Life Data System (www.barcodinglife.org). Molecular Ecology Notes, 7, 355-364.

Rossberg, A.G., Rogers, T. \& McKane, A.J. (2014). Current noise-removal methods can create false signals in ecogenomic data. Proceedings of the Royal Society B, 281, 20140191.

Schloss, P.D., Gevers, D. \& Westcott, S.L. (2011). Reducing the effects of PCR amplification and sequencing artifacts on 16S rRNA-based studies. PLoS ONE, 6, e27310.

Schloss, P.D., Westcott, S.L., Ryabin, T., Hall, J.R., Hartmann, M., Hollister, E.B., Lesniewski, R.A., Oakley, B.B., Parks, D.H., Robinson, C.J., Sahl, J.W., Stres, B., Thallinger, G.G., Van Horn, D.J. \& Weber, C.F. (2009). Introducing mothur: open-source, platform-independent, community-supported software for describing and comparing microbial communities. Applied and Environmental Microbiology, 75, 7537-7541.

Schnell, I.B., Bohmann, K. \& Gilbert, M.T.P. (2015). Tag jumps illuminated - reducing sequence-to-sample misidentifications in metabarcoding studies. Molecular Ecology Resources, 15, 1289-1303.

Shelton, A.O., O’Donnell, J.L., Samhouri, J.F., Lowell, N., Williams, G.D. \& Kelly, R.P. (2016). A framework for inferring biological communities from environmental DNA. Ecological Applications, 26, 1645-1659.

Song, H., Buhay, J.E., Whiting, M.F. \& Crandall, K.A. (2008). Many species in one: DNA barcoding overestimates the number of species when nuclear mitochondrial pseudogenes are coamplified. Proceedings of the National Academy of Sciences of the United States of America, 105, 13486-91.

Taberlet, P., Coissac, E., Pompanon, F., Brochmann, C. \& Willerslev, E. (2012). Towards nextgeneration biodiversity assessment using DNA metabarcoding. Molecular Ecology, 21, 
696

697

698

699

700

701

702

703

704

705

706

707

708

709

710

711

712

713

714

715

716

717

718

719

720

721

722

723

724

725

726

2045-50.

Tang, M., Hardman, C.J., Ji, Y., Meng, G., Liu, S., Tan, M., Yang, S., Moss, E.D., Wang, J., Yang, C., Bruce, C., Nevard, T., Potts, S.G., Zhou, X. \& Yu, D.W. (2015). High-throughput monitoring of wild bee diversity and abundance via mitogenomics. Methods in Ecology and Evolution, 6, 1034-1043.

de Vargas, C., Audic, S., Henry, N., Decelle, J., Mahé, F., Logares, R., Lara, E., Berney, C., Le Bescot, N., Probert, I., Carmichael, M., Poulain, J., Romac, S., Colin, S., Aury, J.-M., Bittner, L., Chaffron, S., Dunthorn, M., Engelen, S., Flegontova, O., Guidi, L., Horák, A., Jaillon, O., Lima-Mendez, G., Lukeš, J., Malviya, S., Morard, R., Mulot, M., Scalco, E., Siano, R., Vincent, F., Zingone, A., Dimier, C., Picheral, M., Searson, S., Kandels-Lewis, S., Acinas, S.G., Bork, P., Bowler, C., Gorsky, G., Grimsley, N., Hingamp, P., Iudicone, D., Not, F., Ogata, H., Pesant, S., Raes, J., Sieracki, M.E., Speich, S., Stemmann, L., Sunagawa, S., Weissenbach, J., Wincker, P. \& Karsenti, E. (2015). Eukaryotic plankton diversity in the sunlit ocean. Science, 348, 1261605.

Williams, S. \& Knowlton, N. (2001). Mitochondrial pseudogenes are pervasive and often insidious in the snapping shrimp genus Alpheus. Molecular Biology and Evolution, 18, 1484-1493.

Zaiko, A., Martinez, J.L., Schmidt-Petersen, J., Ribicic, D., Samuiloviene, A. \& Garcia-Vazquez, E. (2015). Metabarcoding approach for the ballast water surveillance - An advantageous solution or an awkward challenge? Marine Pollution Bulletin, 92, 25-34.

Zhan, A., He, S., Brown, E. a., Chain, F.J.J., Therriault, T.W., Abbott, C.L., Heath, D.D., Cristescu, M.E. \& MacIsaac, H.J. (2014). Reproducibility of pyrosequencing data for biodiversity assessment in complex communities. Methods in Ecology and Evolution, 5, 881-890.

Zhan, A. \& MacIsaac, H.J. (2015). Rare biosphere exploration using high-throughput sequencing: research progress and perspectives. Conservation Genetics, 16, 513-522.

Zhou, J., Wu, L., Deng, Y., Zhi, X., Jiang, Y., Tu, Q., Xie, J., Nostrand, J.D. Van, He, Z., Yang, Y., Van Nostrand, J.D., He, Z. \& Yang, Y. (2011). Reproducibility and quantitation of amplicon sequencing-based detection. The ISME Journal, 5, 1303-13.

Zwickl, D.J. (2006). Genetic algorithm approaches for the phylogenetic analysis of large biological sequence datasets under the maximum likelihood criterion. $\mathrm{Ph} . \mathrm{D}$. dissertation, 
727 The University of Texas at Austin.

728

729 
Figure 1 (on next page)

Diagram illustrating the study design

Photo credit: Matthieu Leray 
Tissue subsampling of 34 specimens and DNA extraction
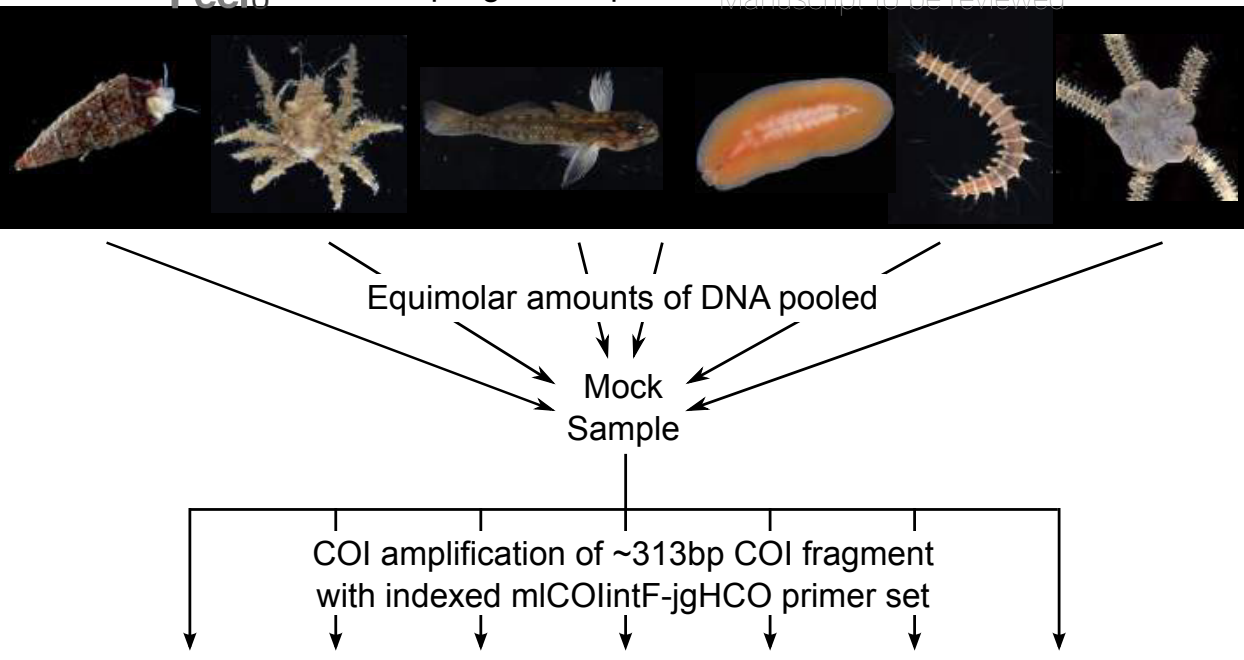

Index Index Index Index Index Index Index
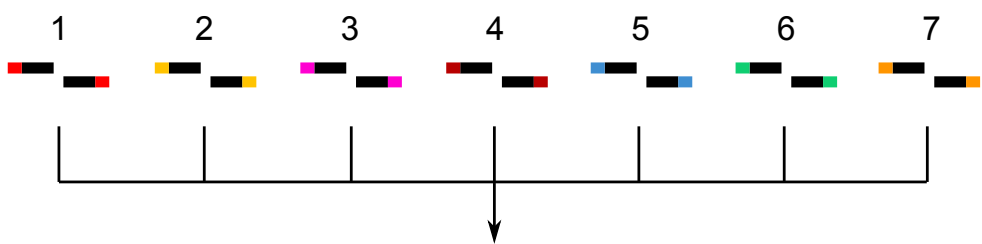

Equimolar pool

of indexed PCR amplicons

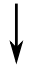

TruSeq library preparation

Illumina MiSeq

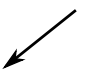$$
\text { q }
$$

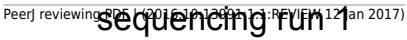

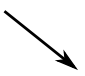

Illumina MiSeq

sequencing run 2 


\section{Figure 2 (on next page)}

Phylogenetic relationships between representative COI sequences (313bp) of 120 OTUs detected in the mock sample.

This is the best tree of 1000 maximum likelihood search replicates computed using the adaptive best tree search analyses implemented in GARLI V2.1 [ (Zwickl 2008) through the GARLI web service (Bazinet et al. 2014) ]. The full tree is provided in Figure S1. Branch tip symbols indicate the method of identification of each OTU. Target: OTU that matched the COI sequence of a species included in the mock sample (referred to as "target OTUs" throughout the manuscript; note that OTUs that did not match any target OTU are referred to as "nontarget OTUs" throughout the manuscript); NCBI/BOLD: OTU that did not match a target OTU but had $>97 \%$ similarity with a reference COI barcode in NCBI or BOLD; SAP: OTU that did not match a target OTU nor a reference COI barcode in NCBI or BOLD but could be confidently assigned to higher a taxonomic level using a Bayesian phylogenetic approach implemented in the Statistical Assignment Package (SAP); Unidentified: OTU that could not be confidently identified to any taxonomic group using the three approaches detailed above. 


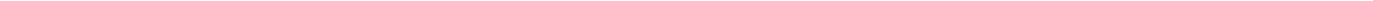




\section{Figure 3 (on next page)}

Rank abundance plot of OTUs detected in the mock sample

Colours indicate the method of identification of each OTU (see Fig. 2 legend). Target: OTU that matched the $\mathrm{COI}$ sequence of a species included in the mock sample (referred to as "target OTUs" throughout the manuscript); NCBI/BOLD: OTU that did not match a target OTU but had $>97 \%$ similarity with a reference COI barcode in NCBI or BOLD; SAP: OTU that did not match a target OTU nor a reference COI barcode in NCBI or BOLD but could be confidently assigned to higher a taxonomic level using a Bayesian phylogenetic approach implemented in the Statistical Assignment Package (SAP); Unidentified: OTU that could not be confidently identified to any taxonomic group using the three approaches detailed above* indicates the presence of an abundant yet unidentified OTU. 


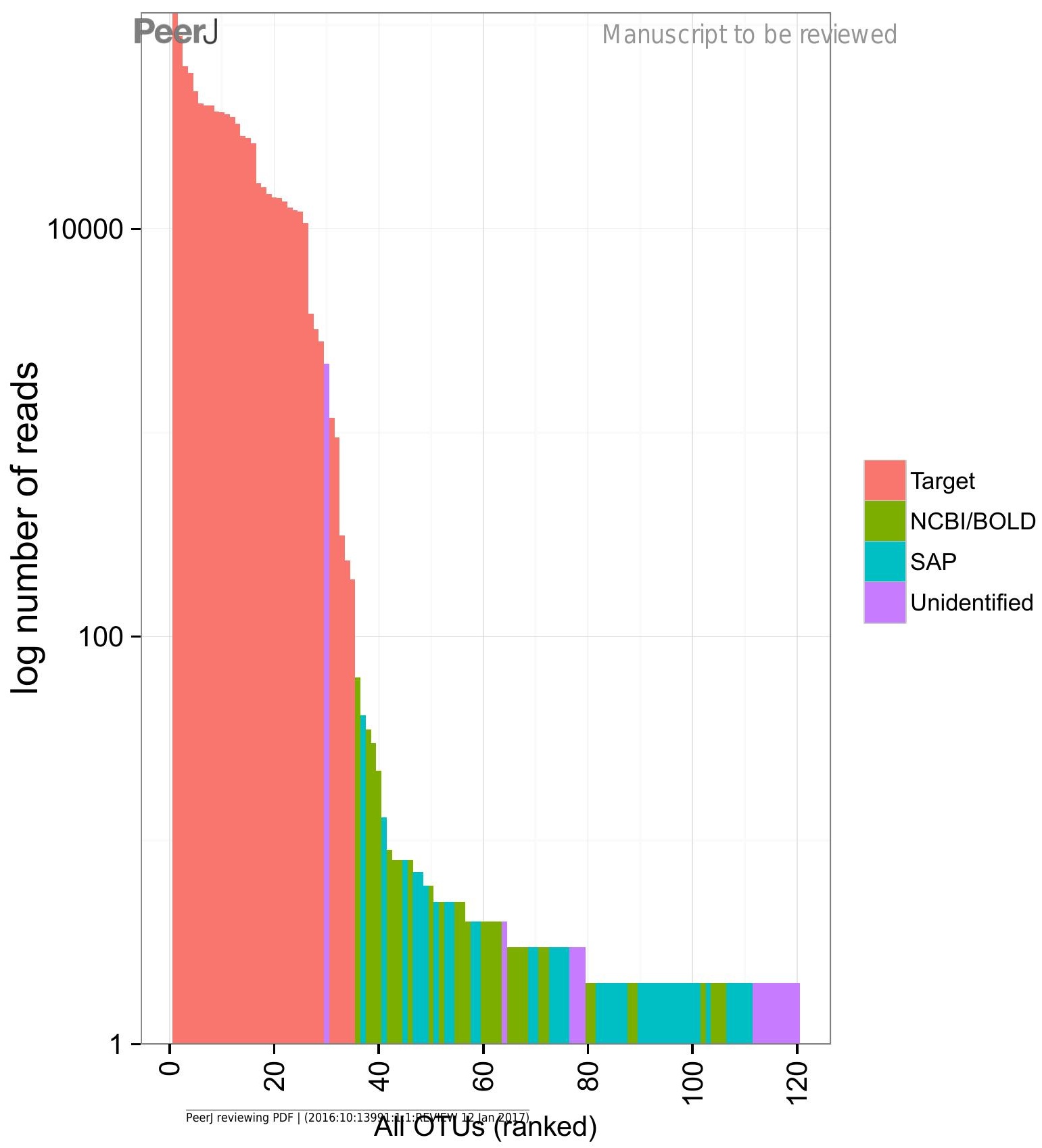


Figure 4 (on next page)

Proportion of reads recovered for each of the target OTUs separately (A) and after combining them per phyla (B)

Mean and standard deviation (SD) were calculated among seven indexed PCRs. The expected proportion of reads was calculated based on the proportion of total genomic DNA pooled (nuclear and mitochondrial DNA). Ranked phyla are as follows: 1) Arthorpoda; 2) Annelida; 3) Chordata; 4) Echinodermata; 5) Mollusca; 6) Platyhelminthes. The identity of the ranked OTUs can be found in table S1. 
Figure $\mathbf{5}$ (on next page)

Reproducibility of non-target OTUs in indexed PCRs as a function of the total number of reads they represent in each sequencing run

"Non-target OTUs" correspond to OTUs that did not match the COI sequence of a species included in the mock sample. Each point represents data for one sequencing run for one nontarget OTU, so that for example, four non-target OTUs found in all seven indexed PCR trials in sequencing run 1 and two non-target OTUs found in all PCR trials in sequencing run 2 had a total number of reads $>10$ for both sequencing runs. 


\section{Figure 6 (on next page)}

Jaccard pairwise dissimilarity among indexed PCRs and Illumina Miseq runs

The effect of rare OTUs on Jaccard was evaluated by sequentially removing low abundance OTUs from the rarefied OTU table (all: full rarefied OTU table; $\geq 2$ : rarefied OTU table without singletons; $\geq 3$ : rarefied OTU table without singletons and doubletons). Jaccard value of 0 indicates that samples have exactly the same OTU composition whereas a value of 1 indicates that samples do have any OTU in common. 


\section{Figure 7 (on next page)}

Bray-Curtis pairwise dissimilarity among indexed PCRs and Illumina Miseq runs

Bray-Curtis value of 0 indicates that samples are identical in terms of OTU composition and abundance of reads whereas a value of 1 indicates that samples do not have any OTUs in common. 


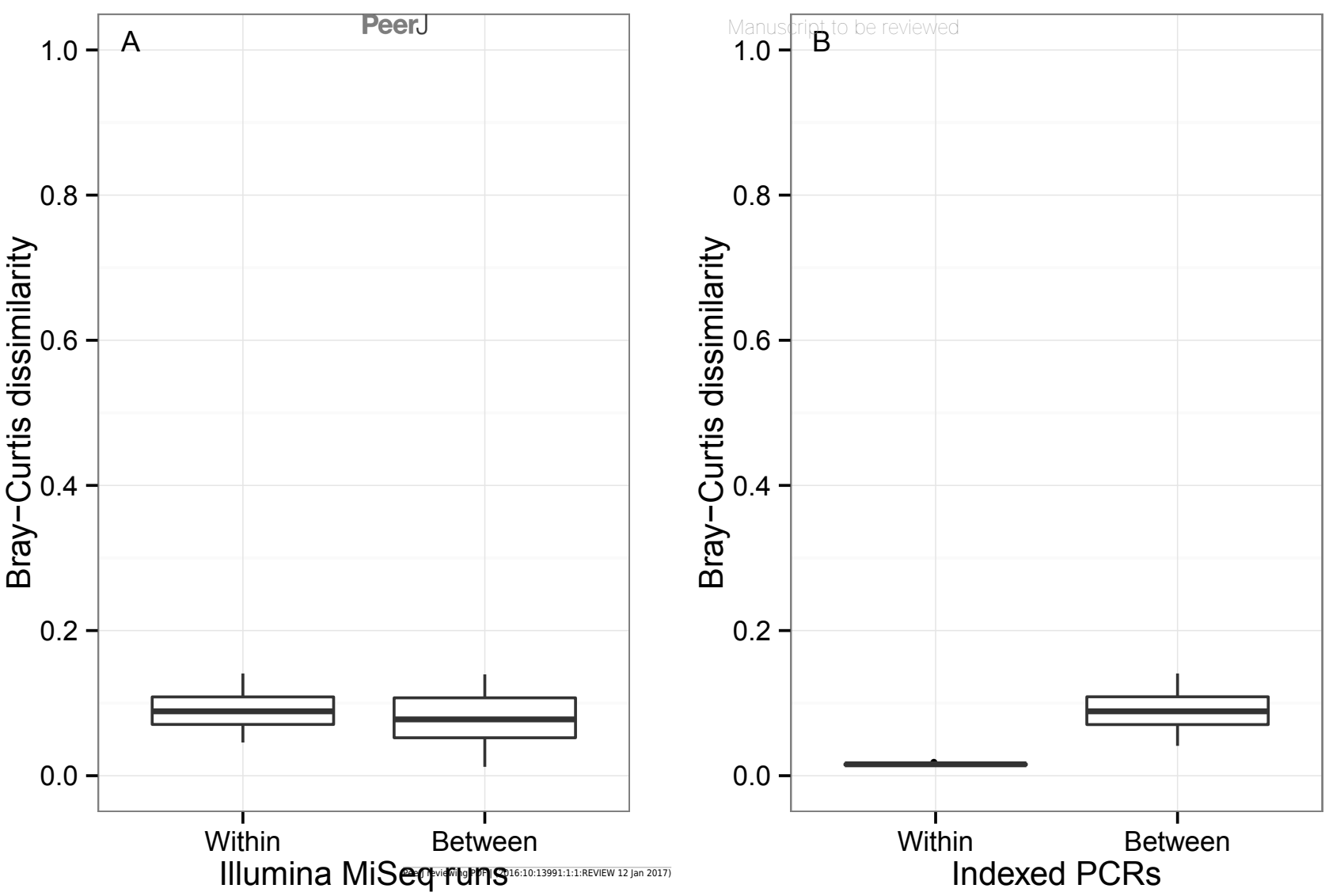


Figure 8 (on next page)

Hierarchical cluster trees constructed using UPGMA with jackknife support to depict similarities in OTU composition between indexed PCRs and Illumina MiSeq runs

Similarities between samples were calculated using the incidence-based Jaccard (A and B) and abundance-based Bray-Curtis (C) indices. All OTUs comprising less than eight sequences were considered rare in B. Branch colors illustrate the level of jackknife support. Red: 75100\%; Orange: 50-75\%; Green: 25-50\%; Blue: 0-25\%. P: Indexed primer pair number. 
Figure 9 (on next page)

Principal Coordinate Analyses (PCOA) depicting Bray-Curtis dissimilarities between samples.

Red crosses represent the score of each OTU in 2-dimensional PCOA. Refer to thefull OTU table in the appendix (Table S2) to link OTU numbers to taxonomic names. 


\section{Table $\mathbf{1}$ (on next page)}

Indexed primers used in this study 
1

\begin{tabular}{ll}
\hline Primer label & Primer sequence (5' - 3') \\
\hline mlCOIintF-Index1 & AGACGCGGWACWGGWTGAACWGTWTAYCCYCC \\
mlCOIintF-Index2 & AGTGTAGGWACWGGWTGAACWGTWTAYCCYCC \\
mlCOIintF-Index3 & ACTAGCGGWACWGGWTGAACWGTWTAYCCYCC \\
mlCOIintF-Index4 & ACAGTCGGWACWGGWTGAACWGTWTAYCCYCC \\
mlCOIintF-Index5 & ATCGACGGWACWGGWTGAACWGTWTAYCCYCC \\
mlCOIintF-Index6 & ATGTCGGGWACWGGWTGAACWGTWTAYCCYCC \\
mlCOIintF-Index7 & ATAGCAGGWACWGGWTGAACWGTWTAYCCYCC \\
jgHCO-Index1 & AGACGCTAIACYTCIGGRTGICCRAARAAYCA \\
jgHCO-Index2 & AGTGTATAIACYTCIGGRTGICCRAARAAYCA \\
jgHCO-Index3 & ACTAGCTAIACYTCIGGRTGICCRAARAAYCA \\
jgHCO-Index4 & ACAGTCTAIACYTCIGGRTGICCRAARAAYCA \\
jgHCO-Index5 & ATCGACTAIACYTCIGGRTGICCRAARAAYCA \\
jgHCO-Index6 & ATGTCGTAIACYTCIGGRTGICCRAARAAYCA \\
jgHCO-Index7 & ATAGCATAIACYTCIGGRTGICCRAARAAYCA \\
&
\end{tabular}




\section{Table 2 (on next page)}

Pairwise dissimilarities calculated among replicates (i.e. pseudo- $\beta$ diversity) to evaluate the effect of random sampling and technical artefacts. 


\begin{tabular}{|c|c|c|}
\hline Pairwise dissimilarity & Description of calculation & $\begin{array}{l}\text { Interpretation of } \\
\text { dissimilarities }\end{array}$ \\
\hline Within indexed primers & $\begin{array}{l}\text { Between communities obtained using identical primer } \\
\text { indices but sequenced in different MiSeq run (i.e. } \\
\text { primer1/run1 vs. primer1/run2; primer2/run1 vs. } \\
\text { primer2/run2) }\end{array}$ & $\begin{array}{l}\text { Result from } \\
\text { random sampling } \\
\text { during sequencing } \\
\text { only }\end{array}$ \\
\hline Between indexed primers & $\begin{array}{l}\text { Between communities obtained using different primer } \\
\text { indices regardless of the sequencing run (i.e. } \\
\text { primer1/run1 vs. primer2/run1; primer2/run1 vs. } \\
\text { primer1/run2) }\end{array}$ & \multirow{3}{*}{$\begin{array}{l}\text { Result from a } \\
\text { combination of } \\
\text { technical artefacts } \\
\text { during PCR (e.g. } \\
\text { amplification bias } \\
\text { caused by primer } \\
\text { indices) and } \\
\text { random sampling } \\
\text { during sequencing }\end{array}$} \\
\hline Within Illumina MiSeq run & $\begin{array}{l}\text { Between communities obtained within the same } \\
\text { MiSeq run regardless of the indexed primer set used } \\
\text { (i.e. primer1/run1 vs. primer } 2 / \text { run } 1 \text {; primer } 2 / \text { run } 1 \text { vs. } \\
\text { primer3/run1) }\end{array}$ & \\
\hline Between Illumina MiSeq run & $\begin{array}{l}\text { Between communities obtained in different MiSeq } \\
\text { runs regardless of the indexed primer set used (i.e. } \\
\text { primer1/run1 vs. primer1/run2; primer1/run1 vs. } \\
\text { primer2/run2) }\end{array}$ & \\
\hline
\end{tabular}




\section{Table 3(on next page)}

Permutational multivariate analysis (PERMANOVA) testing differences in OTU composition between indexed PCRs and Illumina MiSeq runs.

Jaccard considers only the presence-absence of OTUs whereas Bray-Curtis also takes into account differences in abundance of reads between samples. Df: degrees of freedom; SS: sum of squares; F Model: F value by permutation. 
1

2

\begin{tabular}{lllcccc}
\hline & Source of variation & Df & SS & F Model & $\mathrm{R}^{2}$ & P-value \\
\hline \multirow{2}{*}{ Jaccard } & MiSeq Runs & 1 & 0.059 & 1.049 & 0.078 & 0.414 \\
& Indexed PCRs & 6 & 0.357 & 1.059 & 0.474 & 0.367 \\
& Residuals & 6 & 0.337 & & 0.448 & \\
Bray-Curtis & MiSeq Runs & 1 & 0.000 & 4.888 & 0.007 & $<0.001^{* * *}$ \\
& Indexed PCRs & 6 & 0.051 & 106.886 & 0.983 & $<0.001^{* * *}$ \\
& Residuals & 6 & 0.000 & & 0.009 & \\
\hline
\end{tabular}

